OPEN ACCESS

Edited by:

loannis Grammatikakis,

National Institutes of Health $(\mathrm{NIH})$,

United States

Reviewed by:

Bei Liu,

Fourth Military Medical University,

China

Ming-Chuan Hsu,

China Medical University, Taiwan

*Correspondence:

Yan Wang

dryantg@163.com

Rutong Yu

yu.rutong@163.com

†These authors share first authorship

Specialty section: This article was submitted to

RNA,

a section of the journa

Frontiers in Genetics

Received: 22 April 2021 Accepted: 18 August 2021 Published: 15 October 2021

Citation:

Li J, Zhao Z, Wang X, Ma Q, Ji H,

Wang $Y$ and Yu R (2021)

PBX2-Mediated circTLK1 Activates JAK/STAT Signaling to Promote Gliomagenesis via miR-452-5p/SSR1

Axis. Front. Genet. 12:698831.

doi: 10.3389/fgene.2021.698831

\section{PBX2-Mediated circTLK1 Activates JAK/STAT Signaling to Promote Gliomagenesis via miR-452-5p/SSR1 Axis}

\author{
Jing $\mathrm{Li}^{1,2+}$, Zongren Zhao ${ }^{2 t}$, Xiang Wang ${ }^{3}$, Qiong $\mathrm{Ma}^{4}$, Huanhuan $\mathrm{Ji}^{2}$, Yan Wang ${ }^{\text {* }}$ and \\ Rutong $\mathrm{Yu}^{1 *}$
}

\begin{abstract}
1 Institute of Nervous System Diseases, Xuzhou Medical University, Xuzhou, China, ${ }^{2}$ Department of Neurosurgery, Second People's Hospital of Huai'an City, Huai'an Hospital Affiliated to Xuzhou Medical University, Huai'an, China, ${ }^{3}$ Department of Rehabilitation, The Affiliated Xuzhou Rehabilitation Hospital of Xuzhou Medical University, Xuzhou, China, ${ }^{4}$ Jiangsu College of Nursing, Huai'an, China
\end{abstract}

Glioma is considered one of the most lethal brain tumors, as the aggressive blood vessel formation leads to high morbidity and mortality rates. However, the mechanisms underlying the initiation and progression of glioma remain unclear. Here, we aimed to reveal the role of circTLK1 in glioma development. Our results revealed that circTLK1 is highly expressed in glioma tumor tissues and glioma cell lines. We then conducted a series of experiments that showed that circTLK1 was involved in the progression of gliomas. Mechanistically, investigation of the factors downstream of circTLK1 revealed that circTLK1 activated JAK/STAT signaling in glioma cells. Furthermore, AGO2-RIP, RNA-pull down, and luciferase reporter gene assays led to the identification of the novel circTLK1/miR-452-5p/SSR1 axis. Moreover, we investigated the upstream regulator of circTLK1 and found that circTLK1 expression in glioma cells could be regulated by the transcriptional factor PBX2. Taken together, our findings show that circTLK1 mediated by PBX2 activates JAK/STAT signaling to promote glioma progression through the miR-452-5p/SSR1 pathway. These results provide new insights into glioma diagnosis and therapy.

Keywords: circular RNA, circTLK1, miR-452-5p, SSR1, JAK/STAT signaling, glioma, PBX2

\section{INTRODUCTION}

Glioma is one of the most common subtypes of malignant brain cancers and accounts for approximately $27 \%$ of central nervous system tumors (Louis et al., 2016). It is characterized by aggressive blood vessel formation, which leads to high morbidity and mortality (Van Meir et al., 2010; Khasraw et al., 2014). Despite clinical treatment of glioma, such as via surgery, combined chemotherapy, and radiotherapy, which have considerably improved in recent years, outcomes for patients remain unfavorable (Mangiola et al., 2010; Chen and Xu, 2016). Therefore, uncovering the mechanism underlying development of glioma is highly needed.

Circular RNAs (circRNAs) are 200-2,000 bp endogenous RNAs, which are transcribed by RNA polymerase II (Zhang et al., 2018) and forms a covalently closed cyclic structure (Li et al., 2018a). CircRNAs have been found to participate in the progression of multiple pathophysiological 
processes, including cancers (Kristensen et al., 2018; Patop and Kadener, 2018; Di Agostino et al., 2020). They play critical roles in many biological processes of tumorigenesis, such as cell viability (Chen et al., 2017), invasion (Song et al., 2019), migration (Ren et al., 2019), and angiogenesis (Jia et al., 2016). Recently, the role of circRNAs in gliomagenesis progression has also been studied. Yang et al. (2018) found that circRNA FBXW7 suppresses glioma tumorigenesis, Xiong et al. (2019) revealed that circRNA circMAN2B2 mediates glioma progression by regulating S100A8 expression through sponging of miR-1205. Barbagallo et al. (2018) demonstrated that circSMARCA5 affects the migration of glioblastoma multiforme cells by facilitating SRSF1/SRSF3/PTB axis activity. These studies suggest an essential role of circRNA in glioma progression.

CircRNA circTLK1, derived from TLK1 messenger RNA (mRNA), was first identified in a renal cell carcinoma study as an oncogene. CircTLK1 upregulates CBX4 expression to promote renal cell carcinoma development by acting as a molecular sponge for miR-136-5p (Li et al., 2020). Subsequently, $\mathrm{Wu}$ F. et al. (2019) revealed the promotive effects of circTLK1 in ischemic stroke. The function of circTLK1 in myocardial ischemia/reperfusion injury was demonstrated by Song Y.F. et al. (2020). However, whether circTLK1 participates in glioma progression remains unclear.

Here, we aimed to uncover the role of circTLK1 in the initiation and progression of glioma. We found that circTLK1 expression is upregulated in glioma tissues and cell lines. Furthermore, downregulation of circTLK1 inhibited glioma cellular progress and suppressed cell growth in vivo. Investigation of the mechanisms upstream and downstream of circTLK1 revealed that circTLK1 mediated by PBX2 aggravates glioma progression by activating JAK/STAT signaling via the miR-452-5p/SSR1 axis.

\section{MATERIALS AND METHODS}

\section{Clinical Samples}

Thirty pairs of glioma tissues and normal marched tissues were harvested from patients who were diagnosed with glioma in Huai'an Hospital Affiliated to Xuzhou Medical University, Second People's Hospital of Huai'an City from January 2017 to June 2018 and were stored at $-80^{\circ} \mathrm{C}$. These patients did not receive radiotherapy or chemotherapy. Informed consent was obtained from all patients. Sample status was confirmed by two pathologists. This study was approved by the Ethics Committee of the Huai'an Hospital Affiliated to Xuzhou Medical University, Second People's Hospital of Huai'an City (No. HEYLL 201928).

\section{Cell Culture and Treatment}

Glioma cell lines (T98G, LN229, CRT, U251, M059J, and M059K), normal human astrocytes (NHAs), and 293T cells were purchased from the Cell Bank of Shanghai Institutes of Biological Sciences, Chinese Academy of Sciences (Shanghai, China). Cells were maintained in Dulbecco's modified Eagle's medium (DMEM) (Invitrogen, Carlsbad, CA, United States) with 10\% FBS (Gibco,
Waltham, MA, United States) in a $37^{\circ} \mathrm{C}, 5 \% \mathrm{CO}_{2}$ environment. All plasmids, Sh-NC, Sh-circTLK1\#1, Sh-circTLK1\#2, OE-NC, and OE-circTLK1\#1, were purchased from RiboBio (Guangzhou, China). All transfections were performed using Lipofectamine 2000 (Invitrogen, Carlsbad, CA, United States) following the manufacturer's instructions.

\section{Animal Experiment}

Eight-week-old nude mice were separated into three groups $(n=6)$, subcutaneously inoculated with U251 cells $\left(10^{6}\right.$ per mouse), which were preinfected with Sh-NC, Sh-circTLK1\#1, and Sh-circTLK1\#2. After 21 days, tumor samples from mice were harvested. Tumor volumes or end weights were recorded. The animal experiments were approved by the Ethics Committee of the Huai'an Hospital Affiliated to Xuzhou Medical University, Second People's Hospital of Huai'an City.

\section{Quantitative Real-Time Polymerase Chain Reaction}

All RNAs from cells or human tissues were isolated using TRIzol reagent (Invitrogen, Carlsbad, CA, United States). The PARIS $^{\text {TM }}$ Kit (Invitrogen, Carlsbad, CA, United States) was use to perform nuclear and cytoplasmic RNA fractionation. The Reverse Transcription Kit (Invitrogen, Carlsbad, CA, United States) was used to reverse transcribe circRNA, mRNA, and RNA into complementary DNA (cDNA). An internal reference was applied using glyceraldehyde-3-phosphate dehydrogenase (GAPDH). Quantitative real-time PCR (qRT-PCR) assays were conducted using the SYBR-Green Real-Time Kit (Takara, Tokyo, Japan) on a Bio-Rad CFX96 system. The fold expression changes were analyzed using the $2^{-\Delta \Delta \mathrm{Ct}}$ method. All primers used in the current study were as follows: circTLK1, F, 5' -ACAGTTTTGGAAGCTTGGGATCT-3' and R, 5'-TGCTCCCACTTGCAACTCCA3 ${ }^{\prime}$; miR-452-5p, F, 5'-TCGGCAATCATGATGGGCTCCTC- $3^{\prime}$ and R, 5'-CTCA ACTGGTGTCGTGGAGTC-3'; SSR1, F, 5' -AAGAACTACAAA ACCGCCCC-3' and R, 5' ${ }^{\prime}$-ATCCCAGGCTGAGACCCAT- $3^{\prime}$; PBX2, F, 5'-CCCATGTCATGAACCTGCTG-3' and R, 5'-GC GCTGAACTTTCGATGGAT-3'; GAPDH, F, 5' ${ }^{\prime}$-AAGGT CGGAGTCAACGGATTT- $3^{\prime}$ and R, 5' ${ }^{\prime}$-ACCAGAGTTAAAAG CAGCCCTG-3'.

\section{Fluorescence in situ Hybridization Assay}

The Cy3-labeled circTLK1 probe was synthesized and commercially obtained from RiboBio (Guangzhou, China) to localize circTLK1 expression in NHAs. A fluorescence in situ hybridization kit (Geneseed, Guangzhou, China) was used to perform fluorescence in situ hybridization (FISH) assay following the manufacturer's instructions. The cells were imaged using a fluorescence microscope (Leica, Wetzlar, Germany).

\section{Western Blot}

All proteins were isolated using radioimmunoprecipitation assay lysis buffer (Beyotime, Shanghai, China). Bicinchoninic acid (BCA) (Beyotime, Shanghai, China) was used to quantify 
the proteins. The proteins were separated using $10 \%$ sodium dodecyl sulfate-polyacrylamide gel electrophoresis (SDS-PAGE) and transferred onto a polyvinylidene difluoride membrane (EMD Millipore, Billerica, MA, United States). The membranes were blocked with $5 \%$ skim milk at $37^{\circ} \mathrm{C}$ for $90 \mathrm{~min}$. Next, membranes were incubated at $4^{\circ} \mathrm{C}$ overnight with primary antibodies as follows: JAK1 (CST; 1:1,000; 29261S), p-JAK1 (CST; 1:1,000; 74129S), STAT1 (CST; 1:1,000; 14994S), p-STAT1 (CST; 1:1,000; 9167S), STAT3 (CST; 1:1,000; 8768S), p-STAT3 (CST; 1:2,000; 9145S), SSR1 (Sigma; $0.4 \mu \mathrm{g} / \mathrm{ml}$; HPA017062), and GAPDH (CST; 1:1,000; 5174S). Subsequently, the membranes were washed with Tris-buffered saline with Tween 20 (TBST) and incubated with secondary antibodies for $2 \mathrm{~h}$. Protein bands were visualized using ECL (Beyotime, Shanghai, China).

\section{Hematoxylin and Eosin Staining}

Mouse tumor tissues were immersed in $10 \%$ neutral formalin for 1 day under sterile conditions. Next, the tumor samples were dehydrated, embedded in paraffin, and cut into 4 - $\mu$ m-thick sections. H\&E staining was performed via a standard procedure.

\section{CCK-8 Assay}

Cell proliferation assays were conducted using a cell counting kit 8 (CCK-8) solution (Dojindo, Kumamoto, Japan). Cells were cultured in a 96-well plate and incubated with the solution for $3 \mathrm{~h}$. Absorbance at $450 \mathrm{~nm}$ was measured using a Thermomax microplate reader (Molecular Devices, CA, United States). All assays were performed at least three times.

\section{Transwell Assay}

Cell migration was measured using a Transwell chamber (Corning Inc., Corning, NY, United States) with an $8.0-\mu \mathrm{m}$ pore size polycarbonate membrane. Cells $\left(10^{5}\right.$ per chamber $)$ were cultured in up-chambers (matrix for invasion assay) for $24 \mathrm{~h}$, after which cells were removed from the chamber. The cells on the reverse side of the chamber were fixed using $4 \%$ paraformaldehyde and then stained with crystal violet. The migrated cells were visualized using an IX71 inverted microscope (Olympus, Tokyo, Japan).

\section{Scratch Wound Healing Assay}

U251 and CRT cells, upon indicated transfections, were seeded in a six-well plate for culturing at $37^{\circ} \mathrm{C}$ in a $5 \% \mathrm{CO}_{2}$ environment. The fine end of a $10-\mu$ l pipette tip was used to create a scratch wound. The cells were imaged using phase-contrast microscopy at the indicated times.

\section{Biotinylated RNA Pull-Down}

Biotinylated circTLK1 probes, miR-452-5p probes, and normal control probes were obtained from RiboBio (Guangzhou, China). Coimmunoprecipitation buffer (Beyotime) was used to lyse the cells. Probe-coated beads were constructed using the coincubating biotinylated probe and C-1 magnetic beads (Life Technologies, CA, United States) for $120 \mathrm{~min}$. The probes were then incubated with cell lysates overnight. TRIzol reagent was used for RNA isolation, and qRT-PCR assays were performed to analyze the RNA complexes.

\section{AGO2-RNA Binding Protein Immunoprecipitation}

RIP assays were performed using a Magna RIP RNA-binding protein immunoprecipitation kit (Millipore, Germany) with antiAGO2 and anti-IgG following the manufacturer's instructions. The complexes were evaluated using qRT-PCR assays.

\section{Chromatin Immunoprecipitation}

First, glycine was used to terminate the crosslinking reaction in cells with $1 \%$ formaldehyde. Subsequently, the bound RNA-protein was subjected to sonication for fragment production. Next, the antibodies were added, and the proteinated A-Sepharose beads were applied to the immunoprecipitated fractions. The results were analyzed using qRT-PCR analysis.

\section{Luciferase Reporter Assays}

The wild-type (WT) or mutant-type (Mut) sequences of circTLK1, circTLK1 promoter, or SSR1 were subjected to PmirGLO dual-luciferase vectors and then transfected into U251 and 293T cells with NC mimics miR-452-5p mimics or PcDNA 3.1/PcDNA 3.1-PBX2. After $24 \mathrm{~h}$, the luciferase activities in U251 or $293 \mathrm{~T}$ cells were detected using a dual-luciferase reporter assay system (Promega, WI, United States). Firefly luciferase activity was normalized to Renilla luciferase activity.

\section{Statistical Analysis}

Statistical analyses were performed using the SPSS 20 software (SPSS, Chicago, IL, United States). Results are shown as mean \pm SD. Results in two different groups were calculated using unpaired Student's $t$-test, and results among three or more groups were analyzed using one-way ANOVA. All assays were conducted at least thrice. Spearman analysis was used to evaluate the association of expression in the samples. Statistical significance was set at $p<0.05$.

\section{RESULTS}

\section{Measurement of circTLK1 in Gliomas}

First, to uncover the role of circTLK1 in glioma, we assessed circTLK1 expression levels in 30 pairs of human glioma tissues. It was found that circTLK1 expression in glioma tumors was markedly higher than in normal matched tissues (Figure 1A). As shown in Figure 1B, circTLK1 expressed in T98G, LN229, CRT, U251, M059J, and M059K cells was higher than that in NHAs, and circTLK1 was abundantly expressed in U251 and CRT cells. Furthermore, we assessed the circRNA characteristics of circTLK1. The linear TLK1 (mTLK1) expression was significantly downregulated in U251 and CRT cells treated with RNase R; however, the expression of circTLK1 remained stable (Figure 1C). Subsequently, circTLK1 was found to be more stable than mTLK1 in U251 cells treated with the transcription inhibitor actinomycin D (Figure 1D). Random hexamer and oligo (dT)18 primers were used to amplify the TLK1 RNAs. As shown in Figure 1E, TLK1 mRNA expression was significantly upregulated but not that of the circular RNA. Moreover, nuclearcytoplasmic fractionation assays (Figure 1F) and FISH analysis 
A

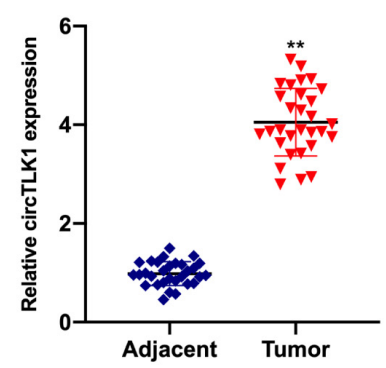

D

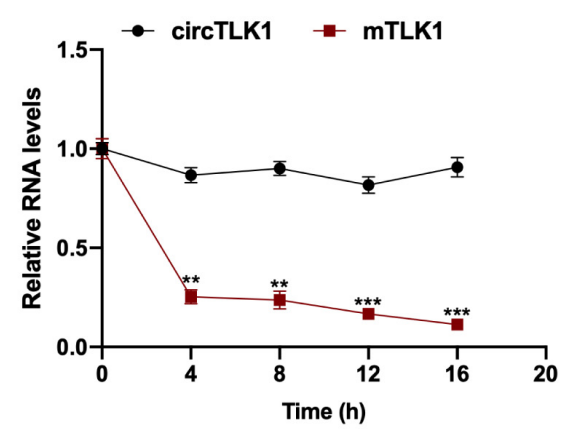

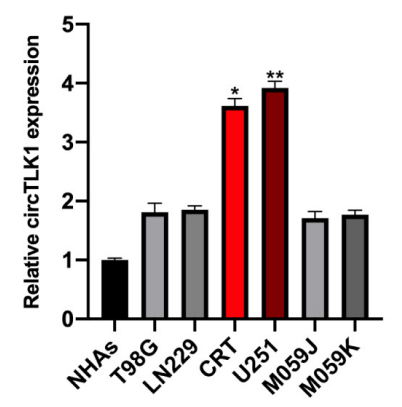

E

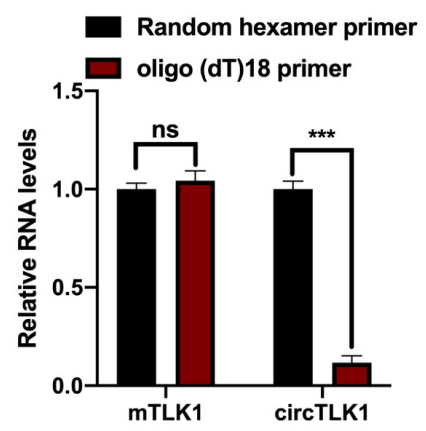

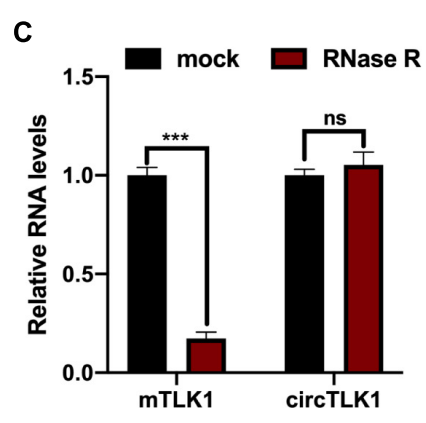

$\mathbf{F}$

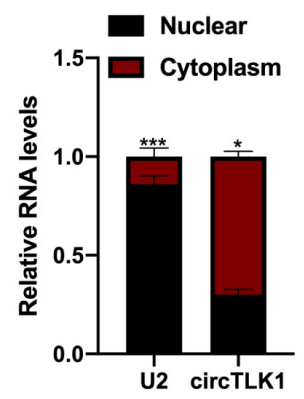

G

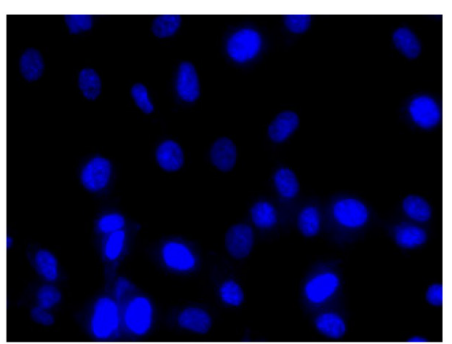

DAPI

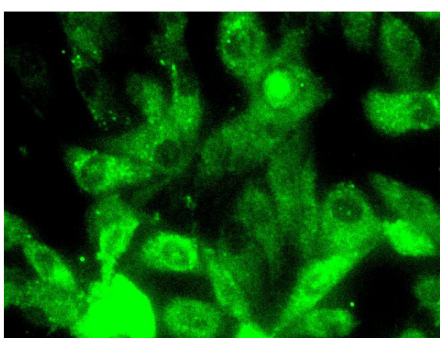

circTLK1

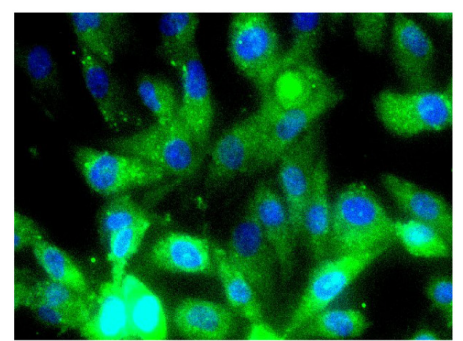

Merge

FIGURE 1 | Measurement of circTLK1 in gliomas. (A) Determination of the circTLK1 expression in 30 pairs of glioma tumor tissues and matched normal tissues using qRT-PCR. (B) qRT-PCR was used to measure circTLK1 expression in glioma cells T98G, LN229, CRT, U251, M059J, M059K, and normal human astrocytes NHAs. (C) Relative RNA levels were analyzed using RT-qPCR; the mock group was used for internal normalization. (D) U251 cells were treated with the transcription inhibitor actinomycin D to evaluate mTLK1 and circTLK1 stability. (E) Relative RNA levels analyzed using RT-qPCR in U251 cells; random hexamer primers were used for internal normalization. (F) The level of circTLK1 in the nuclear and cytoplasm of NHAs was measured using a nuclear-cytoplasmic fractionation assay. (G) FISH analysis was conducted to detect the distribution of circTLK1 in NHAs. ${ }^{*} p<0.05,{ }^{* *} p<0.01,{ }^{* * *} p<0.001$.

(Figure 1G) revealed that circTLK1 was primarily distributed in the cytoplasm. These findings suggests that circTLK1 may be involved in glioma development.

\section{Downregulated circTLK1 Inhibits Glioma Cell Proliferation, Migration, and Invasion}

Here, we aimed to uncover the biological function of circTLK1 in glioma progression. Sh-NC, Sh-circTLK1\#1, and Sh-circTLK1\#2 were constructed and transfected into U251 and CRT cells. The relative expression of circTLK1 in U251 and CRT cells was measured (Figure 2A), and it was found that downregulated
circTLK1 significantly inhibited cell proliferation (Figure 2B). Furthermore, our results showed that downregulated circTLK1 suppressed glioma cell migration and invasion (Figures 2C-E). Taken together, the results show that circTLK1 was involved in glioma progression by mediating increased cell viability, migration, and invasion of glioma cells in vitro.

\section{Downregulated circTLK1 Inhibits Glioma Cell Growth in vivo}

Next, we evaluated the function of circTLK1 in a xenograft nude mouse model. U251 cells $\left(10^{6}\right.$ per mouse), which were 


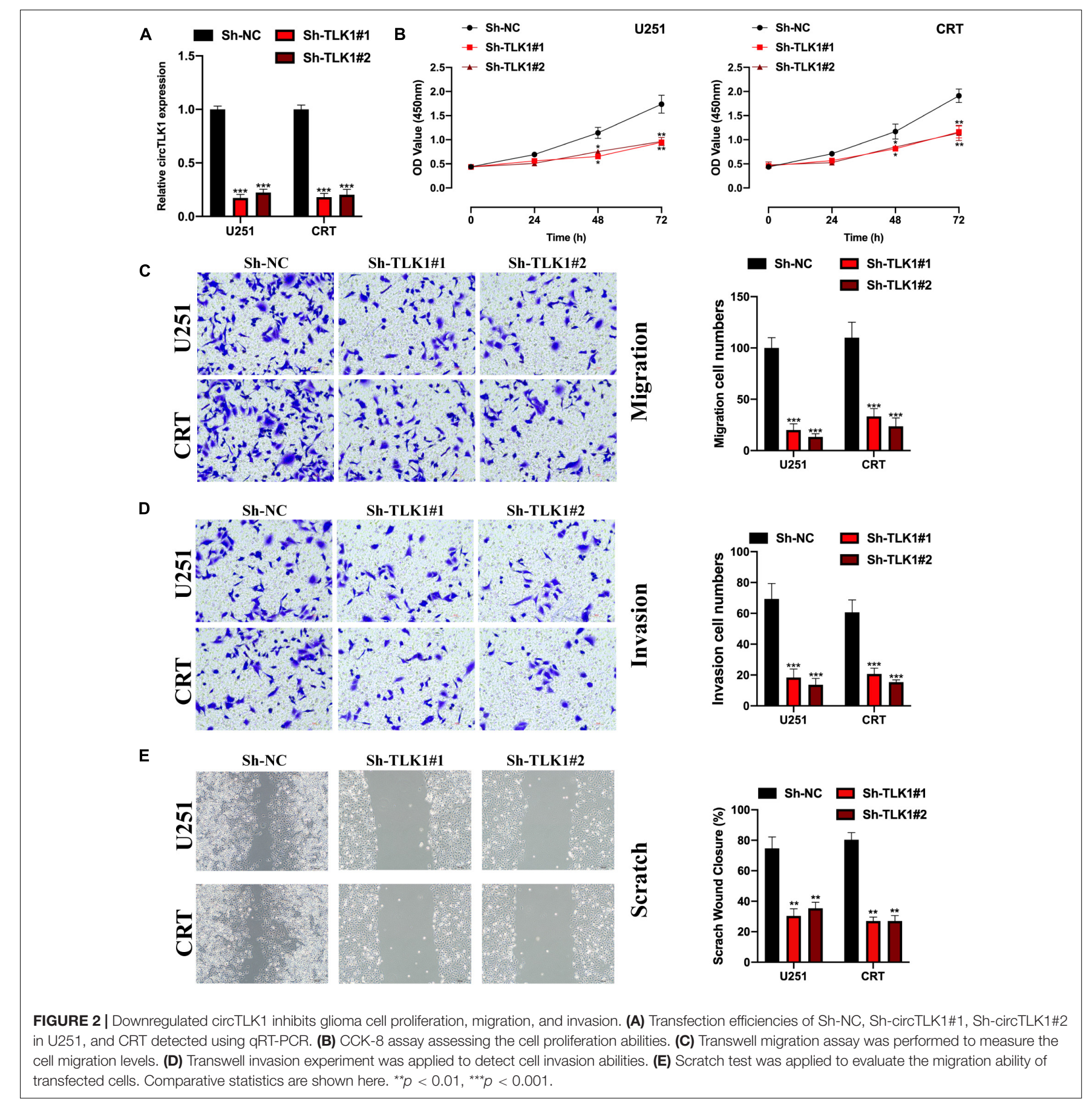

preinfected with Sh-NC, Sh-circTLK1\#1, and Sh-circTLK1\#2, were injected into nude mice. As shown in Figure 3A, representative images of subcutaneous tumors indicated that knockdown of circTLK1 suppressed tumor growth in vivo. Tumor weights and volumes were recorded, and comparative statistics were analyzed (Figures 3B,C). HE staining assays were performed, and the expression of proliferation biomarker Ki67 was assessed in mouse tumor tissues by immunohistochemistry (IHC) staining. The level of Ki67 was obviously decreased in circTLK1 knockdown tumors (Figure 3D). These results indicated that downregulation of circTLK1 suppressed glioma cell growth in vivo.

\section{circTLK1 Modulates Gliomagenesis via Activating JAK/STAT Signaling}

Previous studies have suggested that JAK/STAT signaling plays a role in glioma progression (Tu et al., 2011; Zhang et al., 2019; Swiatek-Machado and Kaminska, 2020). To explore whether circTLK1 regulates glioma progression through 
A

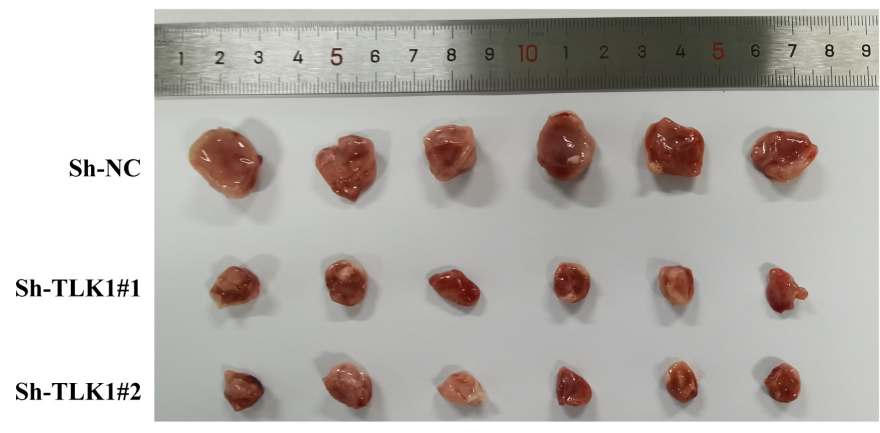

B

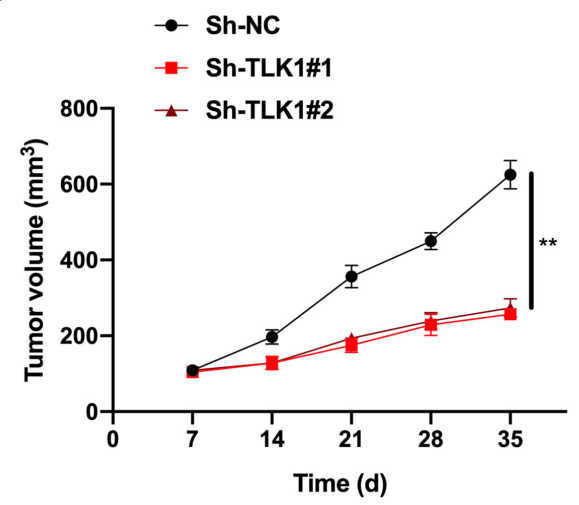

D
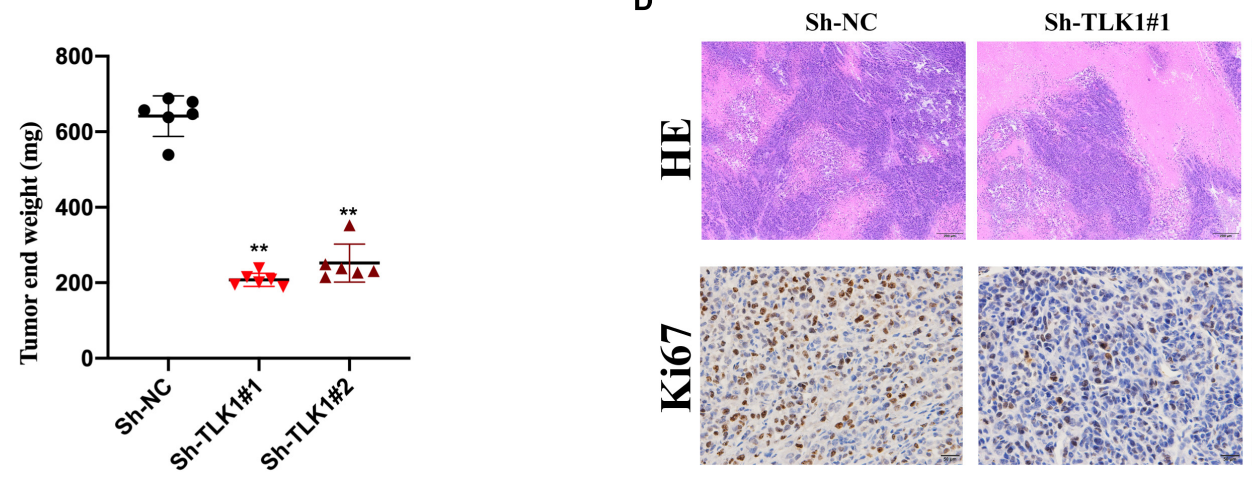

Sh-TLK1\#2

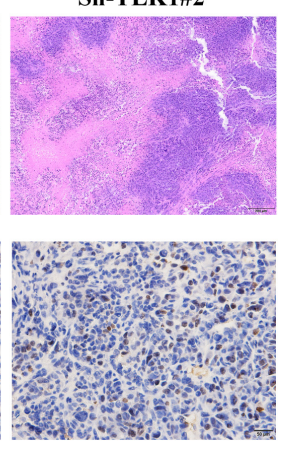

FIGURE 3 | Downregulated circTLK1 inhibits glioma cell growth in vivo. Mice were separated into three groups ( $n=6)$ and then subcutaneously injected with U251 ( $10^{6}$ per mouse) cells preinfected with Sh-NC, Sh-circTLK1\#1, and Sh-circTLK1\#2. (A) Representative image of subcutaneously incubated tumors ( $\left.n=6\right)$. (B) Tumor volumes were recorded every 3 days, and statistical results are presented. (C) Tumor end weights were recorded, and comparative statistics are shown. (D) Tumor histology was assessed using H\&E staining (scale bar, $200 \mu \mathrm{m}$ ), and the proliferation marker Ki67 was detected using IHC staining (scale bar, $50 \mu \mathrm{m}$ ). ${ }^{\star *} p<0.01$.

JAK/STAT signaling, we constructed OE-NC and OE-circTLK1 and transfected them into M059K and M059J cells. CircTLK1 expression was measured in the transfected cells (Figure 4A). Western blot analysis revealed that p-JAK1, p-STAT1, and p-STAT3 expression were significantly increased upon circTLK1 overexpression, while JAK1, STAT1, and STAT3 expression remained unchanged (Figures 4B,C). Moreover, glioma cell models were generated by transfecting OE-NC, OE-circTLK1, and JAK/STAT inhibitor cyt387 as indicated. Overexpression of circTLK1 in M059K and M059J cells promoted cell proliferation (Figures 4D,E), migration (Figures 4F,G,J,K), and invasion (Figures $\mathbf{4 H}, \mathbf{I}$ ), but these phenomena were rescued by treatment with the JAK/STAT inhibitor cyt387. The above results indicate that circTLK1 promotes gliomagenesis by regulating JAK/STAT signaling.

\section{circTLK1 Regulates SSR1 Expression Through Sponging miR-452-5p}

First, we assessed the mRNA binding ability of circTLK1 in U251 cells. AGO2-RNA binding protein immunoprecipitation (AGO2-RIP) assays were performed, and it was found that circTLK1 was abundantly enriched in AGO2 antibody complexes in comparison with anti-IgG (Figure 5A), which showed that
circTLK1 acts as a competitive endogenous RNA (ceRNA) in gliomagenesis. Then, to understand the mechanisms underlying circTLK1 in gliomagenesis, downstream factors were investigated via bioinformatics analysis. Three putative mRNAs were selected, and biotinylated RNA pull-down assays were performed. As shown in Figure 5B, in comparison with bio-NC, miR-452-5p expression was found to be significantly higher than that of other putative mRNAs, suggesting that miR-452-5p might bind to circTLK1. The predicted binding sites between miR-452-5p and circTLK1 (WT or MUT) were synthesized (Figure 5C). The correlation between miR-452-5p and circTLK1 was assessed using a luciferase reporter gene assay. Luciferase activities in 293T and U251 cells preinfected with miR-452-5p mimic and vectors harboring circTLK1 WT sequences were evidently decreased (Figures 5D,E). Subsequently, miR-452-5p expression levels in U251 and CRT cells, infected with Sh-NC, Sh-circTLK1\#1, and Sh-circTLK1\#2, were detected. Knockdown of circTLK1 upregulated miR-452-5p expression (Figure 5F). Moreover, miR452-5p expression was inhibited by circTLK1 (Figure 5G) and downregulated in glioma tumor tissues (Figure $5 \mathbf{H}$ ). The miR452-5p expression in glioma tumor tissues was significantly correlated with circTLK1 (Figure 5I).

Next, we explored the target mRNA of miR-452-5p using the PITA and DIANA-microT databases. The expression of 


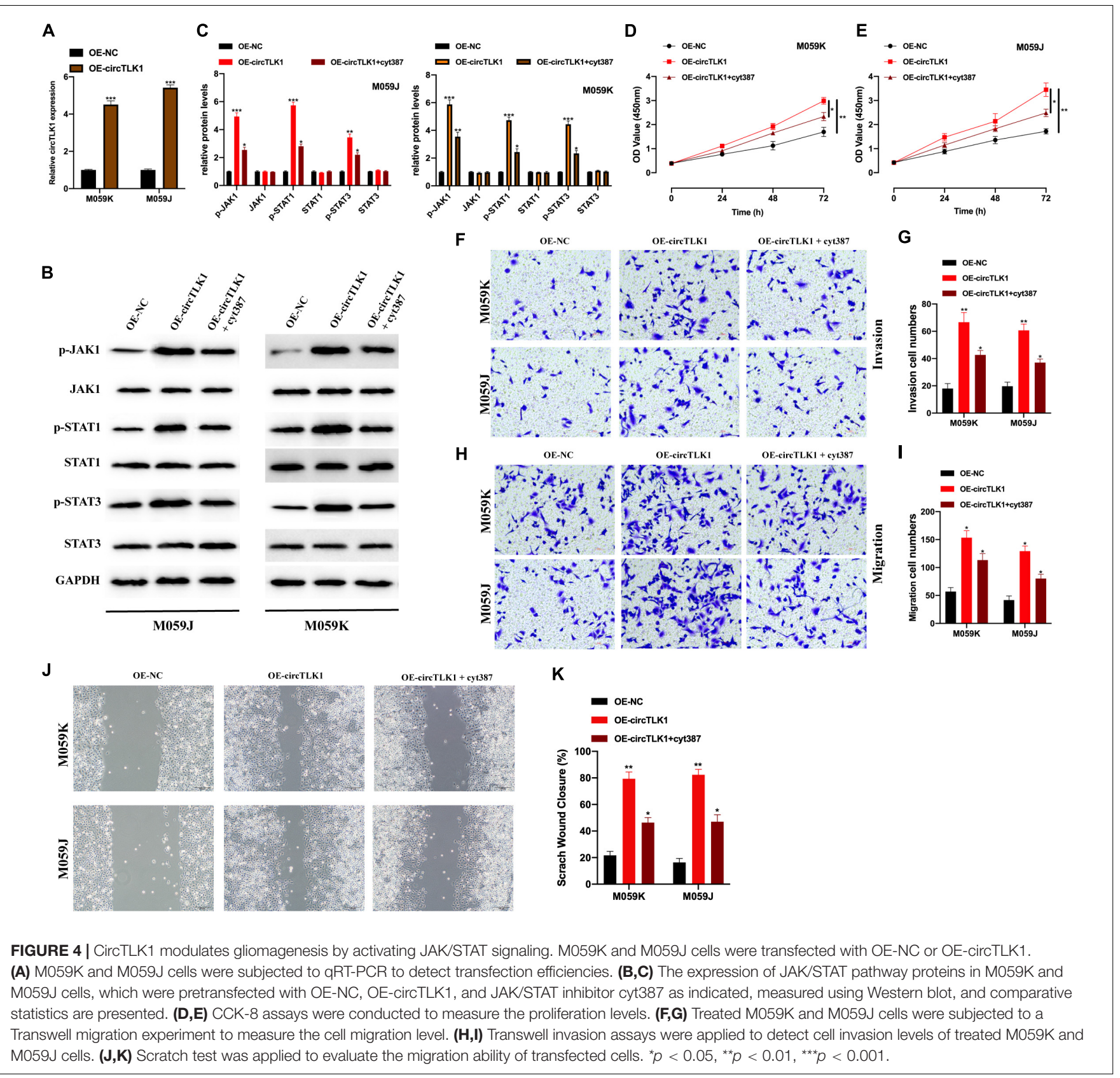

potential mRNA targets in biotinylated probes transfected into U251 cells was assessed; the SSR1 expression level was significantly higher in these cells than in the other groups (Figure 6A). The binding sites between SSR1 (WT or MUT) and miR-452-5p are presented in Figure 6B. Subsequently, we found that luciferase activity in $293 \mathrm{~T}$ and U251 cells preinfected with miR-452-5p mimic and a vector containing SSR1 WT sequences were markedly decreased (Figures 6C,D). The SSR1 expression level was significantly inhibited by circTLK1 knockdown but reversed by miR-452-5p inhibition (Figures 6E-G). This indicated that SSR1 was a downstream target of miR-452-5p and positively mediated by circTLK1. Furthermore, we found that SSR1 expression in glioma tumor tissues was downregulated and correlated with miR-452-5p or circTLK1 expression (Figures 6H-J).

\section{circTLK1 Promotes Gliomagenesis via miR-452-5p/SSR1/JAK/STAT Pathway}

We further explored whether circTLK1 mediates JAK/STAT signaling via the miR-452-5p/SSR1 axis. For this purpose, we constructed glioma knockdown cell models and evaluated the expression of SSR1, and JAK/STAT pathway proteins in these cell models were measured (Figures 7A,B). Subsequently, the biological functions of each cell model were assessed. It was found that overexpression of SSR1 inhibited the suppressive 

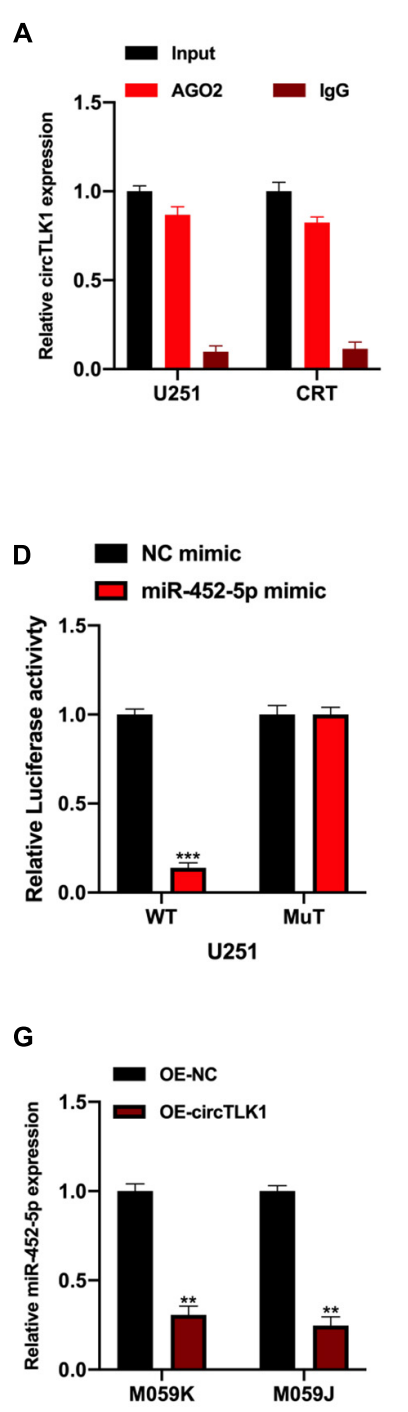

B Biotinylated RNA pull-down

\section{C}

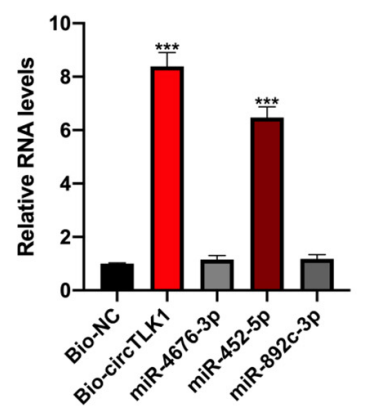

U251
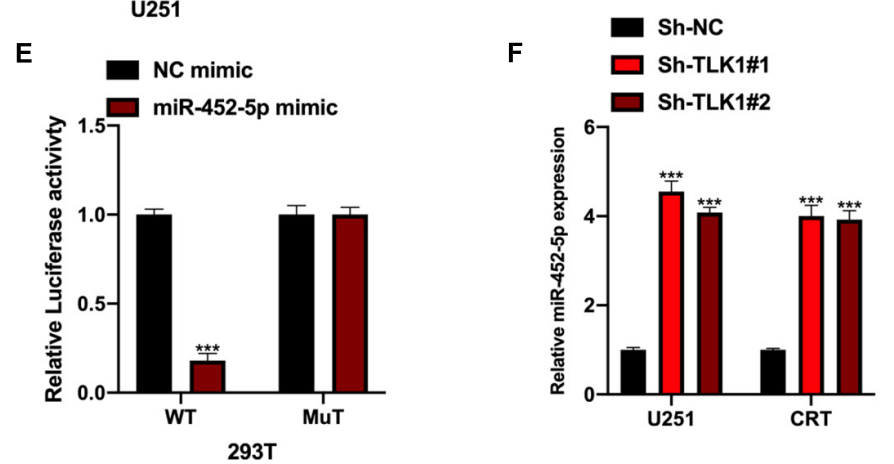

circTLK1 WT: 5` agagaguauagccaAAACAGUa 3,

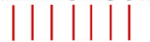

miR-452-5p : 3' agucaaaggagacgUUUGUCAa 5'

circTLK1 MUT: 5' agagaguauagccaUUUGUCAa 3'
H

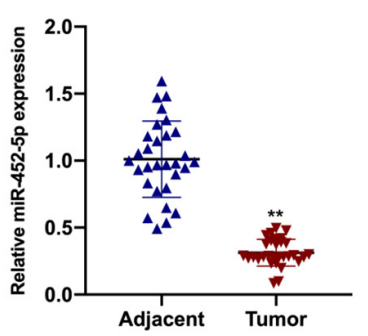

I



FIGURE 5 | CircTLK1 sponges to miR-452-5p. ENCORI database (http://starbase.sysu.edu.cn/index.php) with CLIP data, strict stringency ( $\geq 5$ ), and degradome data, medium stringency $(\geq 2)$ (A) mRNA binding ability of circTLK1 was detected using AGO2-RIP assays in U251 cells. (B) qRT-PCR was performed to assess relative RNA expressions in U251 cells. (C) The binding sites between miR-452-5p and circTLK1 (WT or MUT). (D,E) Relative luciferase activities in NC mimic, miR-452-5p mimic, and reporter vectors containing circTLK1 WT and Mut sequences transfected into 293T and U251 cells were measured. (F) Relative miR-452-5p expression in U251 and CRT cells pretreated with Sh-NC, Sh-circTLK1\#1, and Sh-circTLK1\#2 was measured using qRT-PCR. (G) miR-452-5p expression in U251 and CRT cells pretreated with OE-NC and OE-circTLK1 was evaluated using qRT-PCR. (H) The miR-452-5p expression level in glioma tissues was detected using qRT-PCR. (I) The circTLK1 and miR-452-5p expression association was calculated using Spearman analysis. ${ }^{* *} p<0.01,{ }^{* * *} p<0.001$.

effects of Sh-circTLK1 on cell proliferation (Figure 7C), migration (Figures 7D,E,H,I), and invasion (Figures 7F,G), but these phenomena were rescued by treatment with the JAK/STAT inhibitor cyt387. This suggested that circTLK1/miR452-5p/SSR1 participates in glioma progression by mediating JAK/STAT signaling.

\section{circTLK1 Expression Is Mediated by PBX2}

CircRNA expression could be regulated by transcriptional factors (Wang et al., 2018; Wu L. et al., 2019; Lv et al., 2020), which is an important mechanism in circRNA functional patterns. Here, we further investigated the mechanisms upstream of circTLK1 in gliomagenesis. Using the JASPAR ${ }^{1}$ dataset, we identified three transcriptional regulators (ZNF460. ZNF135, and PBX2) that may mediate circTLK1 expression. Subsequently, we constructed dysregulation cell models of each regulator and measured the expression levels of circTLK1 in these cells. As shown in Figures 8A-C, circTLK1 expression was upregulated in pre-Bcell leukemia transcription factor 2 (PBX2)-overexpressing cells and downregulated in PBX2 knockdown cells. Furthermore,

\footnotetext{
${ }^{1}$ http://jaspar.genereg.net/
} 


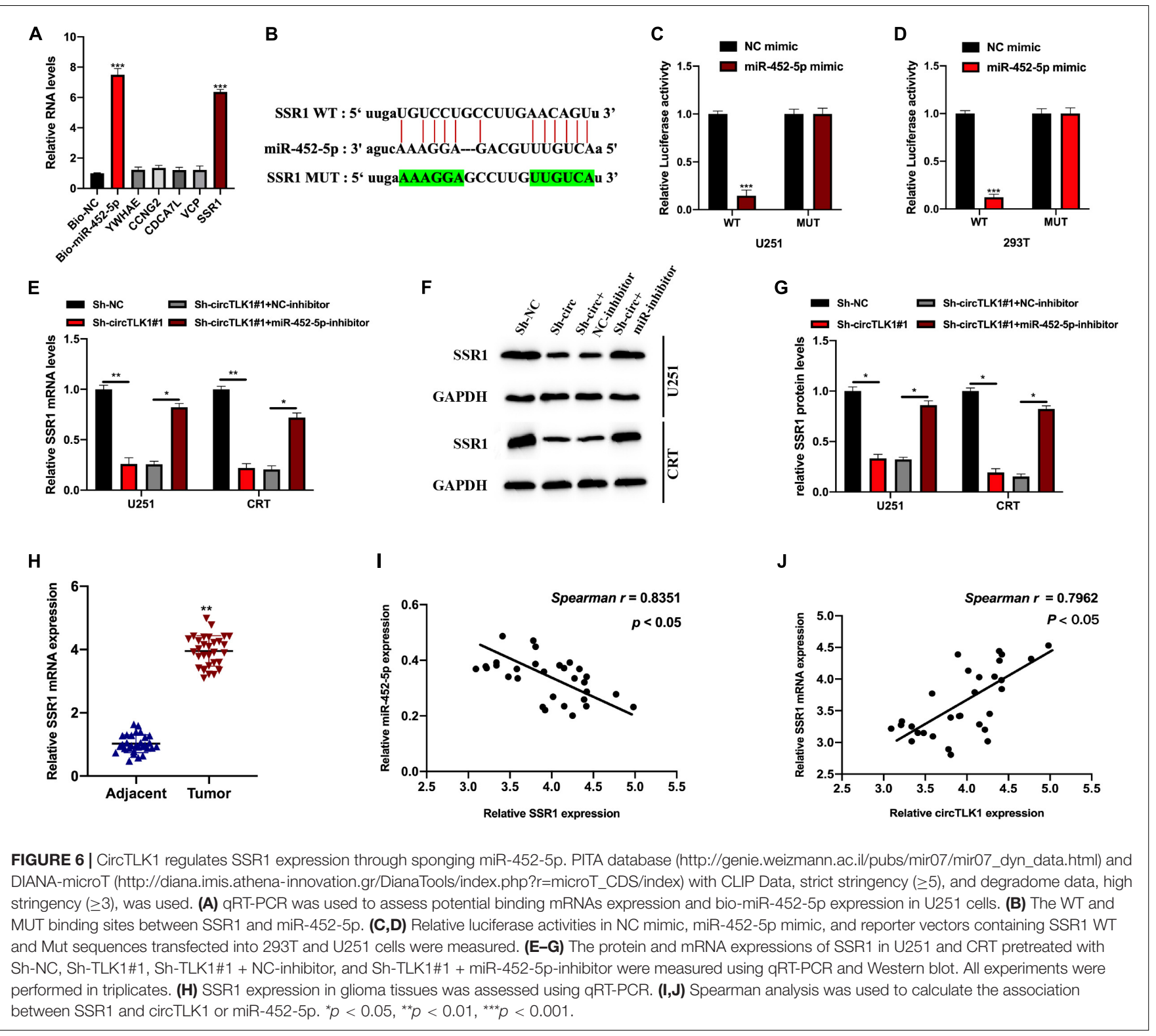

chromatin immunoprecipitation (ChIP) assay results showed that TLK1 was markedly enriched after treatment with antiPBX2 antibody as to isotype control (Figure 8D). Our results suggest that $\mathrm{PBX} 2$ might be at functional upstream regulator of circTLK1 in glioma cells. The predicted binding sequences between the TLK1 promoter and PBX2 were obtained from the JASPAR dataset (Figures 8E,F). The interaction between the TLK1 promoter and PBX2 was assessed using a luciferase reporter assay. As shown in Figures 8G,H, PBX2 overexpression promoted the activity of the wild type (WT) of the TLK promoter, while this phenomenon was attenuated in each mutant type (Mut) of the TLK promoter. Furthermore, the promotive effect of PBX2 overexpression on the TLK1 promoter was completely abrogated when the site 1 and 2 binding sequences were mutated. Our results indicated that circTLK1 expression in glioma cells could be mediated by the transcriptional regulator PBX2.

\section{DISCUSSION}

The findings of this study revealed that circTLK1 mediated by $\mathrm{PBX} 2$ regulated JAK/STAT signaling to promote glioma development by facilitating miR-452-5p/SSR1. First, it was found that that circTLK1 was expressed at higher levels in glioma tissues than in matched normal tissues. The circular RNA characteristic of circTLK1 was assessed. CircTLK1 is abundantly expressed in glioma cells. These results suggest that circTLK1 may be involved in glioma development.

Subsequently, the biological functions of circTLK1 in glioma cell lines were assessed. It was found that circTLK1 knockdown suppressed glioma cell progression by performing CCK-8, Transwell, and scratch assays. Then, animal models were constructed, and our results suggested that downregulated circTLK1 suppressed tumor cell growth in vivo. We noticed that 


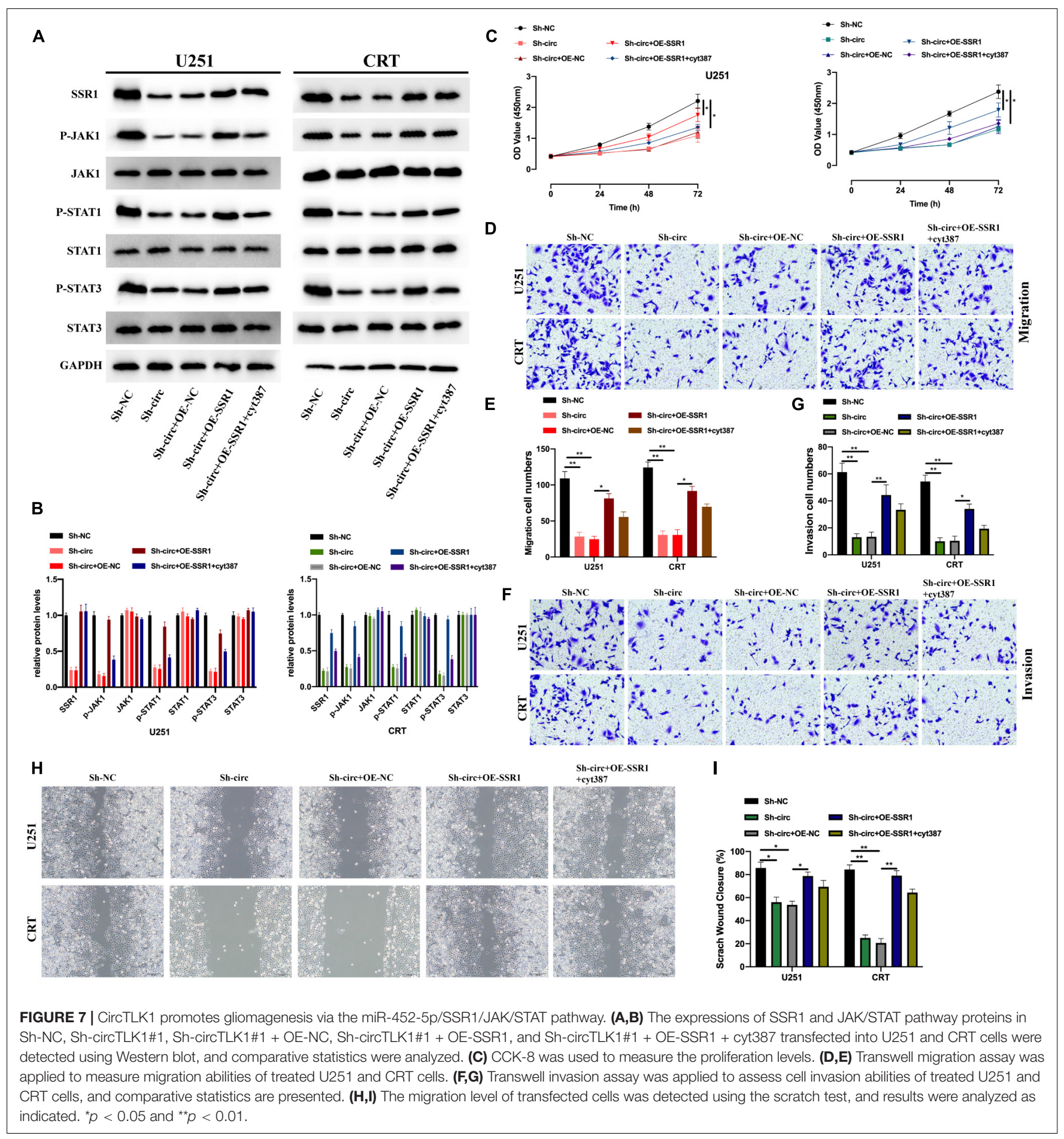

JAK/STAT signaling plays a crucial role in glioma progression, and we presumed that circTLK1 plays a role in mediating JAK/STAT signaling in glioma progression. The expressions of p-JAK1, p-STAT1, and p-STAT3 in circTLK1-overexpressing cells were upregulated, suggesting that JAK/STAT signaling was activated by circTLK1. Moreover, the promotive effects of circTLK1 on glioma cellular progress were rescued by treatment with the JAK/STAT signaling inhibitor cyt387. Our results imply that circTLK1 participates in glioma progression by activating JAK/STAT signaling.

Previous studies have demonstrated that miR-452-5p participates in multiple biological processes, such as colorectal cancer (Yan et al., 2020b), gastric cancer (Zhu et al., 2020), hepatocellular cancer (Yang et al., 2020), prostate cancer (Song X. et al., 2020), and renal cancer (Zhai et al., 2018). However, the role of miR-452-5p in gliomas has not been previously reported. 


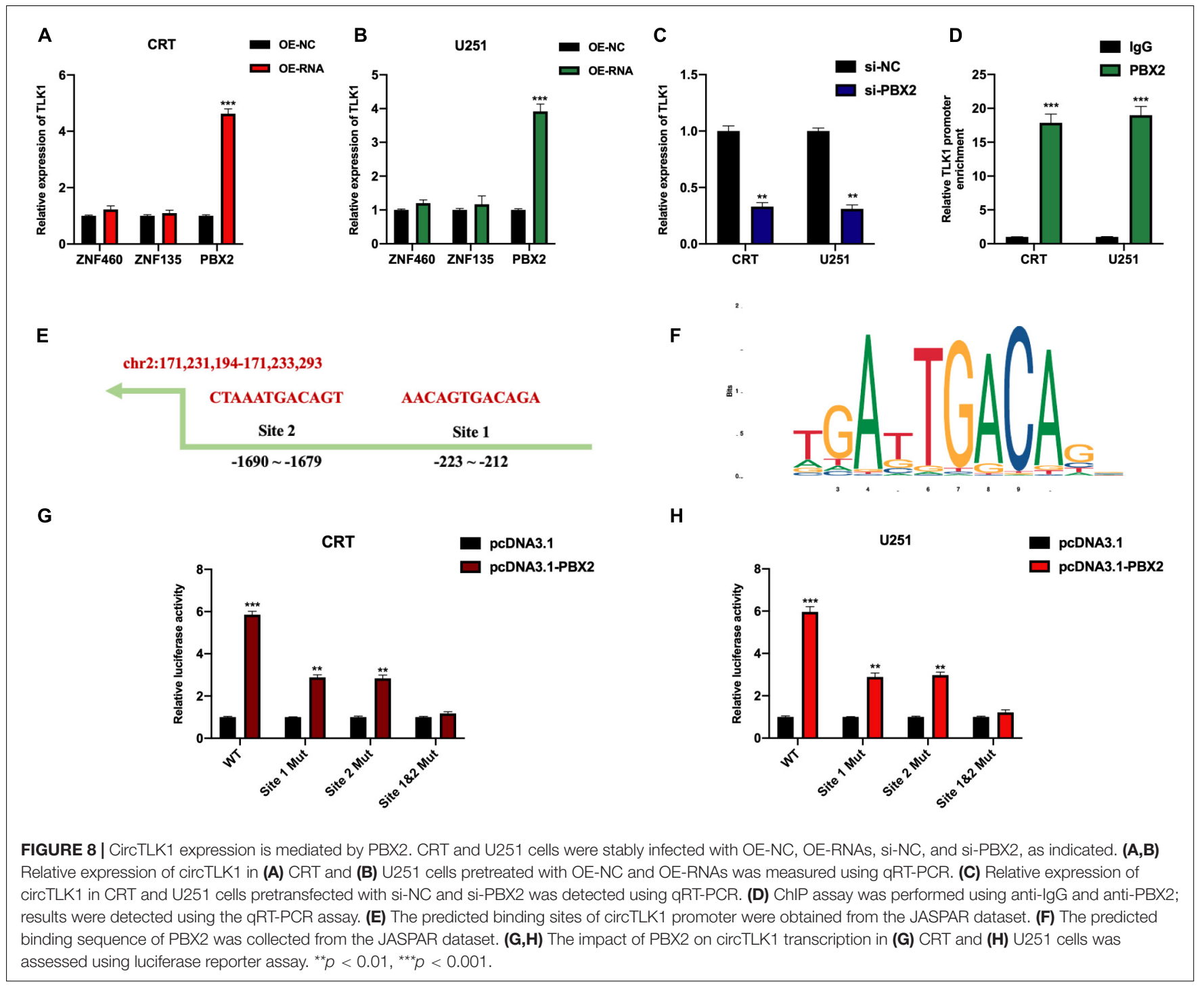

Our results revealed that circTLK1 sponges miR-452-5p and negatively mediates miR-452-5p expression. Signal sequence receptor subunit 1 (SSR1) was found to be a downstream target of miR-452-5p in glioma cells. SSR1 was found to be associated with hypopharyngeal squamous cell carcinoma (Yan et al., 2020a) and breast cancer (Funakoshi et al., 2019). However, whether SSR1 participates in glioma progression remains unclear. Our results revealed that miR-452-5p directly targeted SSR1 and suppressed its expression in glioma cells. Furthermore, JAK/STAT signaling was found to be mediated by the circTLK1/miR-452-5p/SSR1 axis.

The expression of circRNAs can be regulated by transcription factors. Lv et al. (2020) demonstrated that circ-MMP2 expression in lung adenocarcinoma cells is induced by FOXM1 and that the transcription factor c-FOS could bind to the promoter region of circPVT1 and promote circPVT1 expression in nonsmall cell lung cancer cells (Li et al., 2018b). Wang et al. (2018) demonstrated that circ-4099 expression in intervertebral disk degradation is regulated by TNF- $\alpha$-induced GRP78.
Transcription factor-induced expression is an important pattern of circRNAs in various biological processes. In this study, we have partially revealed the downstream mechanisms affected by circTLK1 in gliomagenesis, and we further investigated the upstream regulator of circTLK1. By utilizing the JASPAR dataset, ChIP, and luciferase reporter assays, we identified that PBX2 could bind to the circTLK1 promoter and mediate circTLK1 expression in glioma cells.

Although we partially demonstrated the existence of the novel PBX2/circTLK1/miR-452-5p/SSR1 axis in glioma progression, this will require extensive investigation. Our clinical results require additional samples for further confirmation. Moreover, the relationship between SSR1 and JAK/STAT signaling needs to be verified.

In conclusion, we partially elucidated the role of circTLK1 in glioma progression. CircTLK1 mediated by PBX2 regulates JAK/STAT signaling to promote glioma development via the miR-452-5p/SSR1 axis. Our results provide novel diagnostic and therapeutic targets for treating glioma. 


\section{DATA AVAILABILITY STATEMENT}

The original contributions presented in the study are included in the article/supplementary material, further inquiries can be directed to the corresponding author/s.

\section{ETHICS STATEMENT}

The studies involving human participants were reviewed and approved by the Ethics Committee of the Huai'an Hospital Affiliated to Xuzhou Medical University. The patients/participants provided their written informed consent to participate in this study. The animal study was reviewed

\section{REFERENCES}

Barbagallo, D., Caponnetto, A., Cirnigliaro, M., Brex, D., Barbagallo, C., D'Angeli, F., et al. (2018). CircSMARCA5 inhibits migration of glioblastoma multiforme cells by regulating a molecular axis involving splicing factors SRSF1/SRSF3/PTB. Int. J. Mol. Sci. 19:480. doi: 10.3390/ijms19020480

Chen, G., Shi, Y., Zhang, Y., and Sun, J. (2017). CircRNA_100782 regulates pancreatic carcinoma proliferation through the IL6-STAT3 pathway. Onco Targets Ther. 10, 5783-5794. doi: 10.2147/ott.s150678

Chen, Y., and Xu, R. (2016). Drug repurposing for glioblastoma based on molecular subtypes. J. Biomed. Inform. 64, 131-138. doi: 10.1016/j.jbi.2016.09.019

Di Agostino, S., Riccioli, A., De Cesaris, P., Fontemaggi, G., Blandino, G., Filippini, A., et al. (2020). Circular RNAs in embryogenesis and cell differentiation with a focus on cancer development. Front. Cell Dev. Biol. 8:389. doi: 10.3389/fcell. 2020.00389

Funakoshi, Y., Wang, Y., Semba, T., Masuda, H., Hout, D., Ueno, N. T., et al. (2019). Comparison of molecular profile in triple-negative inflammatory and non-inflammatory breast cancer not of mesenchymal stem-like subtype. PLoS One 14:e0222336. doi: 10.1371/journal.pone.0222336

Jia, P., Cai, H., Liu, X., Chen, J., Ma, J., Wang, P., et al. (2016). Long noncoding RNA H19 regulates glioma angiogenesis and the biological behavior of glioma-associated endothelial cells by inhibiting microRNA-29a. Cancer Lett. 381, 359-369. doi: 10.1016/j.canlet.2016.08.009

Khasraw, M., Ameratunga, M. S., Grant, R., Wheeler, H., and Pavlakis, N. (2014). Antiangiogenic therapy for high-grade glioma. Cochrane Database Syst. Rev. 9, CD008218.

Kristensen, L. S., Hansen, T. B., Veno, M. T., and Kjems, J. (2018). Circular RNAs in cancer: opportunities and challenges in the field. Oncogene 37, 555-565. doi: 10.1038/onc.2017.361

Li, J., Huang, C., Zou, Y., Ye, J., Yu, J., and Gui, Y. (2020). CircTLK1 promotes the proliferation and metastasis of renal cell carcinoma by sponging miR-136-5p. Mol. Cancer 19:103.

Li, X., Yang, L., and Chen, L. L. (2018a). The biogenesis, functions, and challenges of circular RNAs. Mol. Cell 71, 428-442. doi: 10.1016/j.molcel.2018.06.034

Li, X., Zhang, Z., Jiang, H., Li, Q., Wang, R., Pan, H., et al. (2018b). Circular RNA circPVT1 promotes proliferation and invasion through sponging miR-125b and activating E2F2 signaling in non-small cell lung cancer. Cell. Physiol. Biochem. 51, 2324-2340. doi: 10.1159/000495876

Louis, D. N., Perry, A., Reifenberger, G., von Deimling, A., Figarella-Branger, D., Cavenee, W. K., et al. (2016). The 2016 World Health Organization classification of tumors of the central nervous system: a summary. Acta Neuropathol. 131, 803-820. doi: 10.1007/s00401-016-1545-1

Lv, X., Huang, H., Feng, H., and Wei, Z. (2020). Circ-MMP2 (circ-0039411) induced by FOXM1 promotes the proliferation and migration of lung adenocarcinoma cells in vitro and in vivo. Cell Death Dis. 11:426.

Mangiola, A., Anile, C., Pompucci, A., Capone, G., Rigante, L., and De Bonis, P. (2010). Glioblastoma therapy: going beyond Hercules Columns. Expert Rev. Neurother. 10, 507-514. doi: 10.1586/ern.09.158 and approved by the Ethics Committee of the Huai'an Hospital Affiliated to Xuzhou Medical University. Written informed consent was obtained from the individual(s) for the publication of any potentially identifiable images or data included in this article.

\section{AUTHOR CONTRIBUTIONS}

JL, YW, and RY: conception, design, supervision, resources, manuscript writing, and revising. ZZ and XW: experiments conduction and data analysis. QM and $\mathrm{HJ}$ : data visualization and statistical analysis. All authors contributed to the article and approved the submitted version.

Patop, I. L., and Kadener, S. (2018). circRNAs in cancer. Curr. Opin. Genet. Dev. 48, $121-127$.

Ren, S., Liu, J., Feng, Y., Li, Z., He, L., Li, L., et al. (2019). Knockdown of circDENND4C inhibits glycolysis, migration and invasion by up-regulating miR-200b/c in breast cancer under hypoxia. J. Exp. Clin. Cancer Res. 38:388.

Song, T., Xu, A., Zhang, Z., Gao, F., Zhao, L., Chen, X., et al. (2019). CircRNA hsa_circRNA_101996 increases cervical cancer proliferation and invasion through activating TPX2 expression by restraining miR-8075. J. Cell. Physiol. 234, 14296-14305. doi: 10.1002/jcp.28128

Song, X., Wang, H., Wu, J., and Sun, Y. (2020). Long noncoding RNA SOX2OT knockdown inhibits proliferation and metastasis of prostate cancer cells through modulating the miR-452-5p/HMGB3 axis and inactivating Wnt/betacatenin pathway. Cancer Biother. Radiopharm. 35, 682-695. doi: 10.1089/cbr. 2019.3479

Song, Y.F., Zhao, L., Wang, B. C., Sun, J. J., Hu, J. L., Zhu, X. L., et al. (2020). The circular RNA TLK1 exacerbates myocardial ischemia/reperfusion injury via targeting miR-214/RIPK1 through TNF signaling pathway. Free Radic. Biol. Med. 155, 69-80. doi: 10.1016/j.freeradbiomed.2020.05.013

Swiatek-Machado, K., and Kaminska, B. (2020). STAT signaling in glioma cells. Adv. Exp. Med. Biol. 1202, 203-222. doi: 10.1007/978-3-030-30651-9_10

Tu, Y., Zhong, Y., Fu, J., Cao, Y., Fu, G., Tian, X., et al. (2011). Activation of JAK/STAT signal pathway predicts poor prognosis of patients with gliomas. Med. Oncol. 28, 15-23. doi: 10.1007/s12032-010-9435-1

Van Meir, E. G., Hadjipanayis, C. G., Norden, A. D., Shu, H. K., Wen, P. Y., and Olson, J. J. (2010). Exciting new advances in neuro-oncology: the avenue to a cure for malignant glioma. CA Cancer J. Clin. 60, 166-193. doi: 10.3322/caac. 20069

Wang, H., He, P., Pan, H., Long, J., Wang, J., Li, Z., et al. (2018). Circular RNA circ-4099 is induced by TNF-alpha and regulates ECM synthesis by blocking miR-616-5p inhibition of Sox9 in intervertebral disc degeneration. Exp. Mol. Med. 50, 1-14. doi: 10.1038/s12276-018-0056-7

Wu, F., Han, B., Wu, S., Yang, L., Leng, S., Li, M., et al. (2019). Circular RNA TLK1 aggravates neuronal injury and neurological deficits after ischemic stroke via miR-335-3p/TIPARP. J. Neurosci. 39, 7369-7393. doi: 10.1523/jneurosci.029919.2019

Wu, L., Zhang, M., Qi, L., Zu, X., Li, Y., Liu, L., et al. (2019). ERalpha-mediated alterations in circ_0023642 and miR-490-5p signaling suppress bladder cancer invasion. Cell Death Dis. 10:635.

Xiong, J., Wang, T., Tang, H., Lv, Z., and Liang, P. (2019). Circular RNA circMAN2B2 facilitates glioma progression by regulating the miR-1205/S100A8 axis. J. Cell. Physiol. 234, 22996-23004. doi: 10.1002/jcp.28860

Yan, J., Wang, Z. H., Yan, Y., Luo, H. N., Ren, X. Y., Li, N., et al. (2020a). RP11156L14.1 regulates SSR1 expression by competitively binding to miR548ao3p in hypopharyngeal squamous cell carcinoma. Oncol. Rep. 44, 2080-2092.

Yan, J., Wei, R., Li, H., Dou, Y., and Wang, J. (2020b). miR-452-5p and miR215-5p expression levels in colorectal cancer tissues and their relationship with 
clinicopathological features. Oncol. Lett. 20, 2955-2961. doi: 10.3892/ol.2020. 11845

Yang, W., Ju, H. Y., and Tian, X. F. (2020). Circular RNA-ABCB10 suppresses hepatocellular carcinoma progression through upregulating NRP1/ABL2 via sponging miR-340-5p/miR-452-5p. Eur. Rev. Med. Pharmacol. Sci. 24, 23472357.

Yang, Y., Gao, X., Zhang, M., Yan, S., Sun, C., Xiao, F., et al. (2018). Novel role of FBXW7 circular RNA in repressing glioma tumorigenesis. J. Natl. Cancer Inst. 110, 304-315. doi: 10.1093/jnci/djx166

Zhai, W., Li, S., Zhang, J., Chen, Y., Ma, J., Kong, W., et al. (2018). Sunitinibsuppressed miR-452-5p facilitates renal cancer cell invasion and metastasis through modulating SMAD4/SMAD7 signals. Mol. Cancer 17:157.

Zhang, P., Chen, F. Z., Jia, Q. B., and Hu, D. F. (2019). Upregulation of microRNA-133a and downregulation of connective tissue growth factor suppress cell proliferation, migration, and invasion in human glioma through the JAK/STAT signaling pathway. IUBMB Life 71, 1857-1875. doi: 10.1002/iu b. 2126

Zhang, Z., Yang, T., and Xiao, J. (2018). Circular RNAs: promising biomarkers for human diseases. EBioMedicine 34, 267-274. doi: 10.1016/j.ebiom.2018. 07.036
Zhu, L., Wang, C., Lin, S., and Zong, L. (2020). CircKIAA0907 retards cell growth, cell cycle, and autophagy of gastric cancer in vitro and inhibits tumorigenesis in vivo via the miR-452-5p/KAT6B axis. Med. Sci. Monit. 26:e924160.

Conflict of Interest: The authors declare that the research was conducted in the absence of any commercial or financial relationships that could be construed as a potential conflict of interest.

Publisher's Note: All claims expressed in this article are solely those of the authors and do not necessarily represent those of their affiliated organizations, or those of the publisher, the editors and the reviewers. Any product that may be evaluated in this article, or claim that may be made by its manufacturer, is not guaranteed or endorsed by the publisher.

Copyright (c) $2021 \mathrm{Li}$, Zhao, Wang, Ma, Ji, Wang and Yu. This is an open-access article distributed under the terms of the Creative Commons Attribution License (CC BY). The use, distribution or reproduction in other forums is permitted, provided the original author(s) and the copyright owner(s) are credited and that the original publication in this journal is cited, in accordance with accepted academic practice. No use, distribution or reproduction is permitted which does not comply with these terms. 